proportionately found to have mood disorder. Gender of child did not show any association with the post partum blues in the mother. Conclusion Post partum blues has a higher prevalence of $49 \%$ in our region. In India we need to study both the immediate post partum blues and post partum depression to understand their effects on child health.

\section{REVIEW OF CYCLICAL VOMITING SYNDROME IN A GENERAL PAEDIATRIC HOSPITAL OVER A 12 YEAR PERIOD}

doi:10.1136/archdischild-2012-302724.1686

K Odumusi, MJ Mahony, K Atif, B Jamal, C Obi, AM Murphy. Paediatrics, University Hospital Limerick, Limerick, Ireland

Background and Aims Cyclical vomiting syndrome (CVS) is a chronic disorder characterised by recurrent episodes of vomiting separated by symptom free periods. Aim is to review the epidemiology, history, investigation and treatment of children diagnosed with CVS in Mid Western Regional Hospital Limerick from January 2000 to December 2011.

Methods This is a retrospective study of the cases that met the criteria for the diagnosis of CVS. Cases were identified using the hospital in-patient enquiry scheme (HIPE) data. Each patient's case file was assessed for the review.

Results A total of 89 cases of recurrent vomiting were identified using the HIPE data. 10 cases of CVS were identified. Age of onset ranged from 5 to 13 years. $8(80 \%)$ of patients were female. There was an average of 18 months between the onset of symptoms and establishment of diagnosis. Average duration of vomiting episodes was 5 days and average frequency was 6 weekly. $1(10 \%)$ had a positive family history of CVS while $4(40 \%)$ had a family history of migraine. $3(30 \%)$ had associated migraine. $9(90 \%)$ of patients had abdominal $x$-ray and upper GI contrast study while $5(50 \%)$ had upper GI endoscopy and abdominal ultrasound scan. 3(30\%) had brain MRI and $1(10 \%)$ had laparoscopy and MRCP. $9(90 \%)$ of patients were treated with ondasetron, $8(80 \%)$ with proton pump inhibitors and 3(30\%) with cyclizine during episodes. 2(20\%) required psychiatric input.

Conclusions CVS is a disabling condition with female predominance. There is often a delay in diagnosis and large expenditure on investigations before diagnosis is made.

\section{STUDY OF CHILDREN OF BURN VICTIMS ADMITTED IN A BRAZILIAN TEACHING PUBLIC HOSPITAL}

doi:10.1136/archdischild-2012-302724.1687

'NV Moliterno, ' $F$ Moliterno, 'A Veiga, 'EO Veiga, ${ }^{2} S$ Maciel, ${ }^{2} \mathrm{MP}$ Branco, 'S Stumpf, 'SA Nogueira, 'CC Mochdece, 'AP Perie, ' $\mathrm{G}$ Benvenuti, ${ }^{3} \mathrm{DV}$ Rodrigues. 'Faculdade de Medicina de Petrópolis; ${ }^{2}$ Hospital Alcides Carneiro; ${ }^{3}$ Pediatria, Faculdade de Medicina de Petrópolis, Petrópolis, Brazil

Background and Aims The injuries are among the leading causes of morbidity and mortality of children and adolescents. As burns takes the second place in the world of accidents, it is important to be prevented. The aim of this study is describe data from patients hospitalized in a public pediatric unit with a diagnosis of burns in hospital admission.

Methods Cross sectional analysis of documentary records of patients admitted into the Unit of Pediatrics Teaching Hospital in the mountainous region of the State of Rio de Janeiro from May 2009 to May 2011.

Results A total of 29 patients, five (17.24\%) required intensive care. The mean age was 5.73 years and duration of hospitalization was 18.17 days. Predominated by males (65.51\%). The average age of the ICU was 4.6 years with a predominance of males $(60 \%)$ and length of hospital stay was 12.6 days. The heated liquid is the main causative agent, the mean body surface area affected was $42 \%$. Mechanical ventilation of $40 \%$ of patients and hemodynamic support with vasoactive $80 \%$, the mean sedation was 192 hours and the mean of beginning the diet was 43.2 hours, and is the most used jejunal $(60 \%)$. Three patients made use of hyperbaric therapy, with good resolution.

Conclusions The burns are an important public health problem, efforts are needed to reduce accidents and the large number of victims, because the main form of "treatment" for the burn is still prevention through the application of epidemiological principles awareness campaigns and legislative measures.

\section{HOSPITAL@HOME - AN INNOVATIVE COST EFFECTIVE APPROACH TO PAEDIATRIC CARE IN COMMUNITY}

doi:10.1136/archdischild-2012-302724.1688

'S Jyothi, ${ }^{2} \mathrm{~A}$ Vasudevan, ${ }^{3} \mathrm{M}$ Hubbard, ${ }^{4} \mathrm{C}$ Morris, ${ }^{5} \mathrm{~V}$ Yuvaraj. 'Birmingham Children's Hospital, Birmingham; ${ }^{2}$ Longton Hall Surgery; ${ }^{2}$ University Hospital of North Staffordshire; ${ }^{4}$ Staffordshire and Stoke on Trent Partnership Trust, Stoke on Trent; ${ }^{5}$ The John Kelso Practice, Leek, UK

Background A survey in 2009-10 in our region showed 60\% of the total 5500 admissions were discharged from paediatric admissions unit within 4 hours with no active clinical intervention. In early 2011 Local Health Economy(LHE)group consisting of GPs and Paediatricians devised an innovative approach for existing $\mathrm{H} @ \mathrm{H}$ team to manage children with acute illnesses at home and prevent hospital attendance.

Aims To prevent unnecessary hospital attendances and offer a safe cost-effective alternative to short stay admissions by providing clinical care for children at home.

Methods The new H@H service was promoted by GP practice visits and various educational events by the team before commencing in April 2011. Following assessment by GPs, children who needed observation for their acute illnesses and were appropriate to be managed at home were referred to H@H. The H@H team followed local guidelines for management of these children at home and any deterioration in the clinical condition prompted referral to the PAU. Results Until now H@H has been utilised by 90 of 100 GP practices in our region. 950 children were referred and managed by $\mathrm{H} @ \mathrm{H}$ with no adverse events till date. H@H has crossed the conservative cost effective target of reducing 4 PAU referrals each day with a projected saving of $£ 181,000$ /annum. The feedback has been positive from all service users.

Conclusions The two streams of $\mathrm{H} @ \mathrm{H}$

i. early discharge facility

ii. cost-effective admission avoidance facility have proved successful in our region and is currently being evaluated to be introduced by other regions.

\section{REDUCING REFERRALS TO SECONDARY CARE - AN INNOVATIVE APPROACH}

doi:10.1136/archdischild-2012-302724.1689

${ }^{1} \mathrm{~S}$ Jyothi, ${ }^{2} \mathrm{G}$ Julia, ${ }^{2 \mathrm{H}}$ Jenny, ${ }^{3} \mathrm{~A}$ Vasudevan, ${ }^{4} \mathrm{M}$ Hubbard, ${ }^{5} \mathrm{~V}$ Yuvaraj. ${ }^{1}$ Birmingham Childens Hospital, Birmingham; ${ }^{2}$ Partnership in Paediatrics; ${ }^{3}$ Longton Hall Surgery: ${ }^{4}$ University Hospital of North Staffordshire, Stoke on Trent; ${ }^{5}$ The John Kelso Practice, Leek, UK

Background A recent survey showed that in 2009/10 there were approximately 5,500 paediatric admissions in our region. $62 \%$ of these were children referred by GP and $70 \%$ were discharged within 4 hours without active clinical intervention. NHS Stoke-on-Trent, NHS North Staffordshire and Partners in Paediatrics (PiP) devised an innovative approach between primary and secondary care 
clinicians in line with Department of Health OIPP (Quality, Innovation, Productivity and Prevention) programme.

Aims To improve the quality of referrals and reduce inappropriate referrals to admissions unit and outpatients by educating GPs and nurses to be more competent and confident in management of children.

Methods 10 Masterclasses were run by 8 paediatric consultants/ PIP over a 6 week period in 2011. 125 GPs, 92 nurses (17 nurse practitioners, 28 Practice Nurses, 9 community nurses, 24 health visitors) and 29 others (triage, student nurses) attended these sessions. 8 topics including convulsions, gastro enteritis, respiratory infections and rashes covered the top ten non-elective admissions and outpatient referrals to hospital identified in survey.

Results Feedback showed that participants preferred small informal groups with an interactive format helpful for learning. $80 \%$ of 242 participants who completed evaluation forms found the Master-classes useful or extremely useful.

Conclusions Referral data for acute hospital admissions and outpatients for individual GPs before and after Masterclasses is currently being audited. A reduction of $5-10 \%$ in acute and outpatient referrals by GPs is expected by 2012.We aim to conduct paediatric masterclasses to include all primary care clinicians in the region.

\section{PREVALENCE OF FEBRILE SEIZURES IN CHILDREN ADMITTED TO TEACHING PUBLIC HOSPITAL}

doi:10.1136/archdischild-2012-302724.1690

'A Veiga, ${ }^{2} E 0$ Veiga, 'NV Moliterno, ${ }^{2} F M$ Moliterno, 'SS Cordeiro, 'SA Nogueira, ${ }^{3} \mathrm{~A}$ Siqueira, ${ }^{3}$ Cordebel. 'Paediatrics; ${ }^{2}$ Faculdade de Medicina de Petrópolis; ${ }^{3}$ Hospital Alcides Carneiro, Petrópolis, Brazil

Background and Aims Febrile seizure is the manifestation neuropediatric more frequent, occurring in $2 \%$ and $5 \%$ in childhood. It occurs in children between 6 months and 5 years, associated with fever in the absence of intracranial infection or other cause neurological defined and not preceded by afebrile seizures. It is usually generalized tonic-clonic, short, single, early and not accompanied by neurological phenomena after the crisis. The objective of this study was identify the prevalence of Febrile Convulsion in patients hospitalized for febrile seizures, in January 2006 to December 2010.

Methods Cross-sectional study of patients admitted to the pediatric ward in a teaching hospital in the State of Rio de Janeiro. We analyzed 174 patients admitted with seizures of any etiology.

Results Febrile seizures accounted for $36 \%$ of 174 cases of seizures and $1.11 \%$ of 5667 cases admitted in the five years of the study. The mean age was 2.2 years, ranging in age from 6 months and a maximum of six years. The mean hospital stay was 5.73 days. Males accounted for $68.25 \%$ of cases. Regarding the focus of fever, respiratory tract accounted for nearly $50 \%$ of cases being diagnosed with $23.76 \%$ and $19.04 \%$ of upper respiratory infections to pneumonia.

Conclusion This study confirms the prevalence of the epidemiological literature, predominantly male $(2: 1)$, average age between 6 months and 5 years and the respiratory tract as the main focus for febrile seizures. It is imperative that further studies to evaluate diagnosis, treatment and follow up cases.

\section{THE USE OF FATTY ACID SUPPLEMENTATION IN THE TREATMENT OF ADHD- IS THERE ANY EVIDENCE?}

doi:10.1136/archdischild-2012-302724.1691

1,2S Damodaran, ${ }^{3} \mathrm{G}$ Erumbala, ${ }^{4} \mathrm{D}$ Abraham, ${ }^{1} \mathrm{~L}$ Doddamani. 'Paediatrics, James Paget Hospital, Great Yarmouth; ${ }^{2}$ PICU, Addenbrookes Hospital, Cambridge; ${ }^{3}$ Paediatrics, Milton Keynes General Hospital NHS Foundation Trust, Milton Keynes; ${ }^{4}$ Neonatal Unit, Birmingham Women's Hospital, Birmingham, UK

Attention deficit hyperactivity disorder (ADHD) is the most common behavioural disorder in children. The mainstay of treatment is stimulant drugs. There is significant interest in the role of omega 3 fatty acids in ameliorating ADHD symptoms. We reviewed the evidence from available randomized controlled trials.

Clinical question In a child with ADHD (patient), will supplementation with LCPUFA (omega3) (intervention) improve symptoms (outcome)?

Sources Pubmed, Sumsearch, Ovid and Cochraine library. 7 randomised control trials were analysed.

Summary There was no uniformity in terms of the dose or duration of fatty acids. The criteria used for measuring outcome varied significantly. In the largest study, Sinn etal compared omega 3 with omega 3 and multivitamins and placebo. This study involved the longest treatment period. There was significant improvement in ability to switch and control attention. But, there was no improvement in any other cognitive measures. Richardson et al and Belanger et al reported statistically significant improvements in symptoms with higher doses of omega 3. However both used small sample size. The former study lacked robust inclusion criteria. Of the studies that used low dose regime, Gustafson et al demonstrated significant improvement in cognition, inattention and opposition while Voigt et al failed to show any. Interestingly, Hirayama et al showed improvement in the placebo group.

The current available evidence is not sufficient to support the use of omega 3 fatty acid in the treatment of ADHD. More studies need to be conducted in the future using objective outcome criteria and good sample size.

\section{THE AETIOLOGY OF ACUTE ABDOMINAL PAIN IN CHILDREN 2-12 YEARS OF AGE}

doi:10.1136/archdischild-2012-302724.1692

N Kandamany, MB 0'Neill. Mayo General Hospital, Castlebar, Ireland

Background and Aims Abdominal pain is common in childhood. This study evaluated abdominal pain in children aged 2-12 to determine the frequency of aetiologies.

Methods Patients between 2 and 12 years presenting to the Emergency Department over a 5 month period, with abdominal pain were included. Patients with chronic constipation, previous abdominal pain and previous gastrointestinal surgery were excluded. Data was collected prospectively utilising patient's charts and a pretested proforma on abdominal pain which contained diagnostic tips and prompts. Demographic data, referral source, symptom complex, examination findings, and diagnoses were noted. Patients with a diagnosis of constipation were followed up via telephone to confirm accuracy of diagnosis.

Results One hundred and thirteen patients were assessed .Aetiologies of abdominal pain were appendicitis 26 (23\%), constipation $23(20 \%)$, gastroenteritis $18(16.5 \%)$, mesenteric adenitis 15 (13\%),non specific abdominal pain(viral syndrome) 11 (10\%), urinary tract infection $7(6 \%)$, pneumonia $6(5 \%)$, gastritis 5 (4.4\%),acute asthma 2 (2\%). Eighty four (75\%) patients were referred by their family doctor. Patients diagnosed with constipation were contacted by telephone to confirm the diagnosis, with $18(78 \%)$ agreeing.

Conclusion This study outlines the aetiology of abdominal pain in a paediatric population, three quarters of whom had been assessed by their family doctors prior to referral. Non gastrointestinal aetiologies need to be considered in the assessment.

\section{3 \\ PRIAMRY CARE GIVERS OF INFANTS ON HOME OXYGEN}

doi:10.1136/archdischild-2012-302724.1693

${ }^{1} \mathrm{~T}$ Nzirawa, ${ }^{2 \mathrm{M}}$ Ahmed, ${ }^{2} \mathrm{~A}$ Haque. ${ }^{1}$ Neonatal Medicine; ${ }^{2}$ Paediatrics, Queen's University Hospital, London, UK 